low power $(0.2 \mathrm{~mW})$ and with excellent trapping efficiencies (trap stiffness per unit laser power) of $\sim 2.5 \mathrm{pN} \mathrm{\mu m}^{-1} \mathrm{~mW}^{-1}$ in the

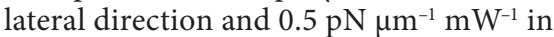
the axial direction, close to the theoretical values. Minimizing laser exposure is important in live biology experiments to reduce optical damage to the specimen.

Interestingly, the authors were also able to use the scheme to allow trapping using a 'non-corrected' high aperture condenser lens, instead of the usual well-corrected microscope objective, thus broadening the design possibilities of optical tweezers.

The most exciting and promising result, however, is successful trapping in a highly turbid medium. In this case, light propagating through a strongly scattering $40-\mu \mathrm{m}$-thick layer of PDMS (polydimethylsiloxane) filled with polystyrene beads ( $5 \mu \mathrm{m}$ in diameter) is used to trap a $1.6 \mu \mathrm{m}$ polystyrene bead suspended in water behind the scattering layer. A supplementary laser holds an identical bead, to be used as the intensity probe, at the exact trapping position. It is important to note that the aberration correction is site-specific, and must be repeated and modified for every target point of the sample.
This constraint currently hinders the application of the method to real biological specimens, as individual particles functioning as sensing probes must be injected and immobilized at controlled sites within the sample, and this may not always be practical. More conveniently, the reference for optimization could be the target to be trapped itself, which should be static and labelled with a fluorophore compatible with near-infrared, nonharmful optical manipulation. Quantum dots would probably be required, which, owing to their improved photostability and brightness over conventional organic dyes, can help in the detection of light from the probe and its differentiation from the background. Furthermore, owing to the long refresh time of the spatial light modulator technology, the optimization procedure is currently too slow to follow time-varying distortions (occurring in ex vivo or in vivo experiments). It is encouraging to hear that the authors are already considering an alternative design to achieve sub-second correction.

There are other less demanding (yet still interesting) applications in which in situ aberration correction should yield significant progress, such as in colloidal studies, data storage and micromachining. For optical tweezers, trapping inside living cells has already been demonstrated ${ }^{9}$ in single-cell environments with small aberrations. The prospect of intact deep manipulation within complex tissues is both challenging and stimulating. Many other biological techniques, including nanosurgery, tissue engineering and advanced microscopy, would also benefit from advances in in situ aberration correction.

Estela Martin-Badosa is at the Department of Applied Physics and Optics, University of Barcelona, Martí i Franquès 1, E-08028 Barcelona, Spain. e-mail:estela.martinb@ub.edu

\section{References}

1. Čižmár, T., Mazilu, M. \& Dholakia, K. Nature Photon. 4, 388-394 (2010)

2. Booth, M. J. Phil. Trans. R. Soc. A 365, 2829-2843 (2007).

3. Girkin, J. M., Poland, S. \& Wright, A. J. Curr. Opin. Biotechnol. 20, 106-110 (2009)

4. Wulf, K. D. et al. Opt. Express 14, 4170-4175 (2006).

5. Jesacher, A. et al. Opt. Express 15, 5801-5808 (2007).

6. Vellekoop, I. M. \& Mosk, A. P. Opt. Lett. 32, 2309-2311 (2007)

7. Vellekoop, I. M., van Putten, E. G., Lagendijk, A. \& Mosk, A. P. Opt. Express 16, 67-80 (2008).

8. Vellekoop, I. M. \& Mosk, A. P. Phys. Rev. Lett. 101, 120601 (2008)

9. Ashkin, A., Schütze, K., Dziedzic, J. M., Euteneuer, U. \&

Schliwa, M. Nature 348, 346-348 (1990).

\title{
LASER FABRICATION
}

\section{Making silicon superhydrophilic}

Although liquids typically flow downwards, the capillary effect - a gravity-defying process exploited by the roots and stems of plants - also allows them to be drawn up through narrow tubes. The phenomenon relies on the adhesion of liquid to the walls of a narrow vessel, pulling the edges of the liquid up to form a concave meniscus. Surface tension then causes the entire column of liquid to be drawn upwards - a process commonly called wicking.

Now, A. Y. Vorobyev and Chunlei Guo from the Institute of Optics at the University of Rochester, USA, have demonstrated that laser pulses can be used to treat the surface of silicon so that it becomes superhydrophilic and exhibits the capillary effect (Opt. Express 18, 6455$6460 ; 2010$ ). The pulsed beam from an amplified Ti:Sapphire femtosecond laser was used to create a $22 \mathrm{~mm} \times 11 \mathrm{~mm}$ array of parallel microgrooves on a $25 \mathrm{~mm} \times 25 \mathrm{~mm} \times 0.65 \mathrm{~mm}$ singlecrystal phosphorus-doped silicon sample.

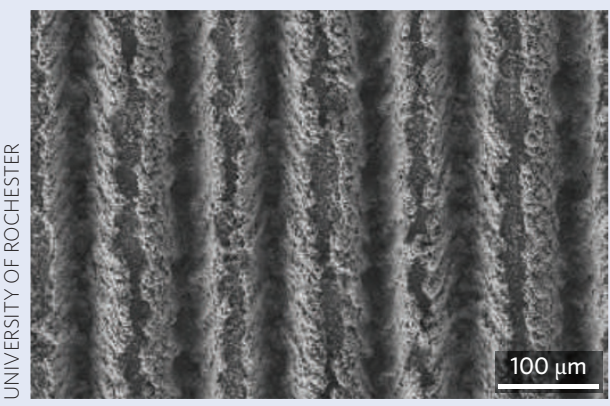

The laser generated $65 \mathrm{fs}$ pulses at a central wavelength of $800 \mathrm{~nm}$ and with $1.5 \mathrm{~mJ}$ of energy per pulse, fabricating each microgroove at a scanning speed of $1 \mathrm{~mm} \mathrm{~s}^{-1}$ and with a focused laser spot diameter of $100 \mu \mathrm{m}$. The resulting grooves had an average depth of $40 \mu \mathrm{m}$.

The researchers tested the wetting properties of the samples by applying 1-5 $\mu \mathrm{L}$ drops of various liquids, including distilled water, acetone and methanol, to their silicon surfaces. Using a camera to capture the spreading dynamics of the liquids at a speed of five frames per second, they were able to compare the wicking behaviours of liquids on both laser-treated and untreated samples of silicon. The results showed that the laser treatment caused the silicon to become superhydrophilic, and that the spreading distance of the liquids, regardless of chemical composition, followed the classical square root of time dependence - a characteristic of the Washburn equation, which governs the motion of liquids in a closed capillary. The same results were achieved when the silicon samples were stood vertically, with the liquid rising upwards against gravity.

Vorobyev and Guo believe that the capillary properties of their ultrafastlaser-treated silicon may find applications in a number of areas, including in microfluidics, lab-on-a-chip technology, biomedicine, and chemical and biological sensors.

SONIA SHAHI 